\title{
Abordagem teórico-experimental das relações de escala e fractalidade da vegetação em áreas sazonalmente alagadas na Floresta Amazônica
}

\author{
Theoretical and experimental approach of scale relations and fractality of \\ vegetation on a seasonally flooded area in the Amazon Forest
}

\author{
Adriano Pereira Guilherme ${ }^{\mathrm{I}}$, Deniz dos Santos Mota ${ }^{\mathrm{II}}$, \\ Iramaia Jorge Cabral de Paulo"II, Sérgio Roberto de Paulo ${ }^{\text {III }}$
}

\begin{abstract}
Resumo
A relação entre a fisiologia e o metabolismo das plantas, assim como os fluxos de energia mediados pela vegetação, é pouco compreendida atualmente. De acordo com a teoria de West, Brown e Enquist (WBE), espera-se que alguns parâmetros de planta, como o diâmetro dos galhos, obedeçam a distribuições do tipo lei de potência. Neste trabalho, buscou-se fazer uma abordagem das relações de escala e fractalidade em duas áreas sazonalmente alagadas da Floresta Amazônica. Especificamente, visou-se responder se há uma relação do tipo lei de potência (livre de escala) para a distribuição dos diâmetros dos troncos nas áreas de estudo, se há uma relação semelhante com respeito à distribuição de diâmetros de galhos em algumas espécies nessas mesmas áreas, e ainda se existe semelhança com a distribuição dos troncos, de tal forma que se possa dizer que a árvore "imita" a floresta em que está contida. Também se apresentou uma forma de medir a fractalidade nas árvores estudadas com base no coeficiente de variação das relações entre diâmetros de galhos de gerações subsequentes $(\beta)$. Observou-se que alguns expoentes diferem dos preditos pela teoria de WBE e que as distribuições de troncos são do tipo lei de potência, mas com variações expressivas de $R^{2}$. Para os galhos, os resultados indicam que os parâmetros realmente seguem leis de potência, mas com variações de $R^{2}$ entre as espécies estudadas. Também se mostrou que pelo menos uma espécie é mais autossimilar (fractal) que outras. Por fim, foi feita uma breve discussão sobre qual o papel desse padrão na adaptação e evolução das plantas.
\end{abstract}

Palavras-chave: Alometria; Lei de potência; Modelo WBE

\begin{abstract}
The relationship between plant physiology and metabolism, as well as energy flows mediated by vegetation, is poorly understood today. According to the West, Brown and Enquist (WBE) theory, some plant parameters, such branch diameters, are expected to obey power law distributions. In this work, we tried to approach the relations of scale and fractality in two seasonally flooded areas of the Amazon Forest. Specifically, the aim was to answer if there is a relationship of power law type (free of scale) for the distribution of the diameters of the trunks in the study areas, if there is a similar relationship with respect to the distribution of the branch diameters in some species in these same areas, and there is still a similarity with the distribution of the trunks, in such a way that we can say that the tree 'imitates' the forest in which it is contained. We also present a way to measure the fractality in the studied trees based on the relationships between the branch diameters of the subsequent generations $(\beta)$ coefficient of variation. It was observed that some exponents differ from those predicted by the WBE theory and that the trunk distributions are of the power law-type, but with expressive variations of $R^{2}$. For the branches, the results indicate that the parameters really follow power laws, but with variations in $R^{2}$ between the studied species. It has also been shown that at least one species is more self-similar (fractal) than others. Finally, there was a brief discussion about what is the role of this pattern in the adaptation and evolution of plants.

Keywords: Allometry; Power-law; WBE model

Físico, Dr., Professor do Instituto de Saúde e Biotecnologia, Universidade Federal do Amazonas, Estrada Coari-Mamiá, 305, CEP 69460-000, Coari (AM), Brasil. adrianopgpg@gmail.com (ORCID: 0000-0001-5203-9917)

Físico, Dr., Professor do Departamento de Física, Instituto de Ciências Exatas, Universidade Federal do Amazonas, Av. General Rodrigo Otávio Jordão Ramos, 1200, CEP 69067-005, Manaus (AM), Brasil. dmota@ufam.edu.br (ORCID: 0000-0003-1578-3012)

III Físico(a), Dr(a)., Professoro(a) do Instituto de Física, Universidade Federal de Mato Grosso, Av. Fernando Correa, s/n, Coxipó, CEP 78060-900, Cuiabá (MT), Brasil. iramaiaj@gmail.com (ORCID: 0000-0001-7164-4290) / sergioufmt@gmail.com (ORCID: 0000-0002-5101-6227)
\end{abstract}




\section{Introdução}

Há décadas a humanidade tem se preocupado em intensidade crescente com o meio ambiente, como se tem percebido pelos últimos relatórios do Painel Intergovernamental sobre Mudanças Climáticas (IPCC) (NAÇÕES UNIDAS, 2014). Nesse contexto, é evidente a grande importância de se compreender em maior profundidade os seres vivos, com bom destaque para o design e metabolismo de ecossistemas, pois são assuntos provavelmente ligados às dinâmicas climáticas nas mais diversas escalas. Especificamente, pode-se perguntar como as plantas evoluíram para buscar maximizar a captação de luz solar, água e nutrientes, ao mesmo tempo em que minimizam a energia gasta para distribuir seiva por todas as suas células? Uma hipótese bastante razoável seria supor que os formatos geométricos e relações que envolvem o metabolismo sejam parte da resposta a tais questões.

É conhecido o fato de que, a despeito das inúmeras diferenças entre os seres vivos, algumas relações envolvendo tamanho, massa e taxas metabólicas parecem seguir padrões aproximadamente universais (RUBNER, 1883; KLEIBER, 1932; BRODY; PROCTER; ASHWORTH, 1934; BARTHOLOMEW, 1981). O estudo dessas relações é um ramo da Biologia chamado Alometria, o qual apresenta, de forma mais frequente, relações envolvendo leis de potência, na forma da Equação (1):

$$
y(x)=a x^{b}
$$

onde: $y$ é a variável dependente; $a$ é uma constante; $x$ é a variável independente; $b$ é o expoente de escala.

Rubner (1883) propôs que a taxa metabólica basal $B$ deveria ser proporcional à superfície $S$ dos animais, resultando em uma relação teórica do tipo $B \sim S \sim M^{2 / 3}$. Tal expoente não se verificou experimentalmente, e uma relação empírica foi encontrada por Kleiber (1932) e corroborada, com o estudo de outras espécies, por Brody, Procter e Ashworth (1934). Nesse caso, o expoente encontrado foi $3 / 4$, e vários outros expoentes múltiplos de $1 / 4$ foram encontrados para outras grandezas (PETERS, 1983; CALDER, 1984; SCHMIDT-NIELSEN, 1984), fato que não teve explicação teórica por algum tempo.

West, Brown e Enquist (1997) elaboraram um modelo (conhecido como modelo WBE) para explicar esses expoentes, mais especificamente o da relação envolvendo a massa e a taxa metabólica basal, baseados em três hipóteses básicas: 1) A rede de distribuição de nutrientes nos organismos mais complexos segue um padrão de ramificação fractal, de tal forma que preencha todo o volume do mesmo, mantendo o modelo de repetição nas sucessivas gerações. Nesse caso, cada galho (ou vaso) ramifica em $n$ galhos menores, o que se repete em todos os níveis (ou geração) $\mathrm{k}$, até os capilares (nível N). 2) As grandezas (como espessura e comprimento) relacionadas à última ramificação da rede, conhecidos por vasos capilares, são invariantes em relação à massa e à espécie. 3) A rede de distribuição foi aperfeiçoada por seleção natural de tal forma que o transporte de recursos gasta o mínimo de energia possível. Em outras palavras, a impedância (resistência ao transporte dos fluidos como sangue ou seiva) é minimizada.

Os resultados do modelo se baseiam principalmente em considerações geométricas. Resumidamente, é suposto que a ramificação da rede conserva a área total de passagem de fluido (Lei de Leonardo). Também se supõe que, para que a rede seja eficiente em termos de distribuição de nutrientes, garantindo que todas as células fiquem próximas a algum capilar, ela deve preencher todo o espaço do organismo. Assim, a ramificação ocorre de tal forma que um grupo de células servidas por um capilar forme o que se chama de volume de serviço, simplificado para uma esfera com raio igual ao comprimento do vaso, volume este que também deve ser conservado no passar de cada geração. Tais considerações resultam finalmente na relação

Ci. Fl., Santa Maria, v. 30, n. 4, p. 1061-1074, out./dez. 2020 
procurada entre a taxa metabólica basal e a massa: $B \propto M^{3 / 4}$. A partir das hipóteses do modelo, os autores também explicaram outras relações alométricas conhecidas com expoentes múltiplos de 1/4 (WEST; ENQUIST; BROWN, 1999), o que não eximiu o modelo de críticas (KOZŁOWSKI; KONARZEWSKI, 2004). Seguindo na linha da hipótese da geometria fractal, propuseram que a distribuição dos diâmetros dos troncos em uma região deveria ser semelhante à distribuição dos diâmetros dos galhos das árvores contidas nessa região, ou seja, as árvores "imitariam" a floresta onde estão (WEST; ENQUIST; BROWN, 2009). Pela segunda hipótese do modelo, era esperado que a distribuição dos diâmetros dos galhos (e, no caso, também dos troncos) resultasse em uma lei de potência de expoente -2 (demonstração em GUILHERME, 2017).

Dessa maneira, neste trabalho, buscou-se fazer uma abordagem das relações de escala e fractalidade com aplicação do modelo WBE em duas áreas sazonalmente alagadas da Floresta Amazônica. Especificamente, visou-se responder da melhor maneira possível às seguintes questões: 1) Há uma relação do tipo lei de potência (livre de escala) para a distribuição dos diâmetros dos troncos nas áreas de estudo? E se há, existe semelhança com outros trabalhos na literatura (universalidade)? 2) Há uma relação semelhante com respeito à distribuição de diâmetros e comprimentos de galhos em algumas espécies nessas mesmas áreas? Se há, existe semelhança com a distribuição dos troncos, de tal forma que se possa afirmar que a árvore "imita" a floresta em que está contida? 3) Como se pode avaliar a medida de fractalidade nas árvores estudadas? E qual o papel, se existe, desse padrão na adaptação e evolução das plantas?

\section{Material e métodos}

\section{Área de estudo}

O bioma Amazônia representa cerca de $30 \%$ de todas as florestas tropicais remanescentes do mundo. De importância reconhecida mundialmente, principalmente por conta de sua larga extensão (4,2 milhões de $\mathrm{km}^{2}$ somente em território brasileiro) e enorme diversidade de ambientes, está presente no território de mais nove países sul-americanos, sendo também a maior floresta tropical do mundo (SERVIÇO FLORESTAL BRASILEIRO, 2010). Por estar em baixas latitudes, recebe uma forte entrada de energia solar. O clima é equatorial, com temperaturas médias muito altas e chuvas abundantes, com precipitação média de $2300 \mathrm{~mm} / \mathrm{ano}$, chegando a $3500 \mathrm{~mm} / \mathrm{ano}$ em algumas regiões. No entanto, existem duas estações razoavelmente bem definidas: uma seca e outra chuvosa (FISCH, 1998).

O estudo foi realizado no município de Coari - AM (região central do estado brasileiro do Amazonas), mais especificamente em uma localidade conhecida por Ilha da Botija (Figura 1), localizada em $3^{\circ} 58^{\prime} 43^{\prime \prime} \mathrm{S}$ e $62^{\circ} 53^{\prime} 60^{\prime \prime} \mathrm{W}$. Foram analisadas duas regiões distintas, uma bastante próxima de um curso de água (região 1) e outra mais alta e distante (região 2), ambas suscetíveis a alagamentos sazonais, sendo que a região 1 permanece alagada por mais tempo. Tais regiões são popularmente denominadas, respectivamente, de igapó e várzea. 


\section{Figura 1 - Local das coletas dos dados. Acima: Localização do município de Coari no estado do Amazonas. Abaixo: Ilha da Botija, da qual os dados foram efetivamente coletados}

Figure 1 - Data collection area. Above: Location of the municipality of Coari in the state of Amazonas, Brazil. Below: Botija Island where data were effectively collected

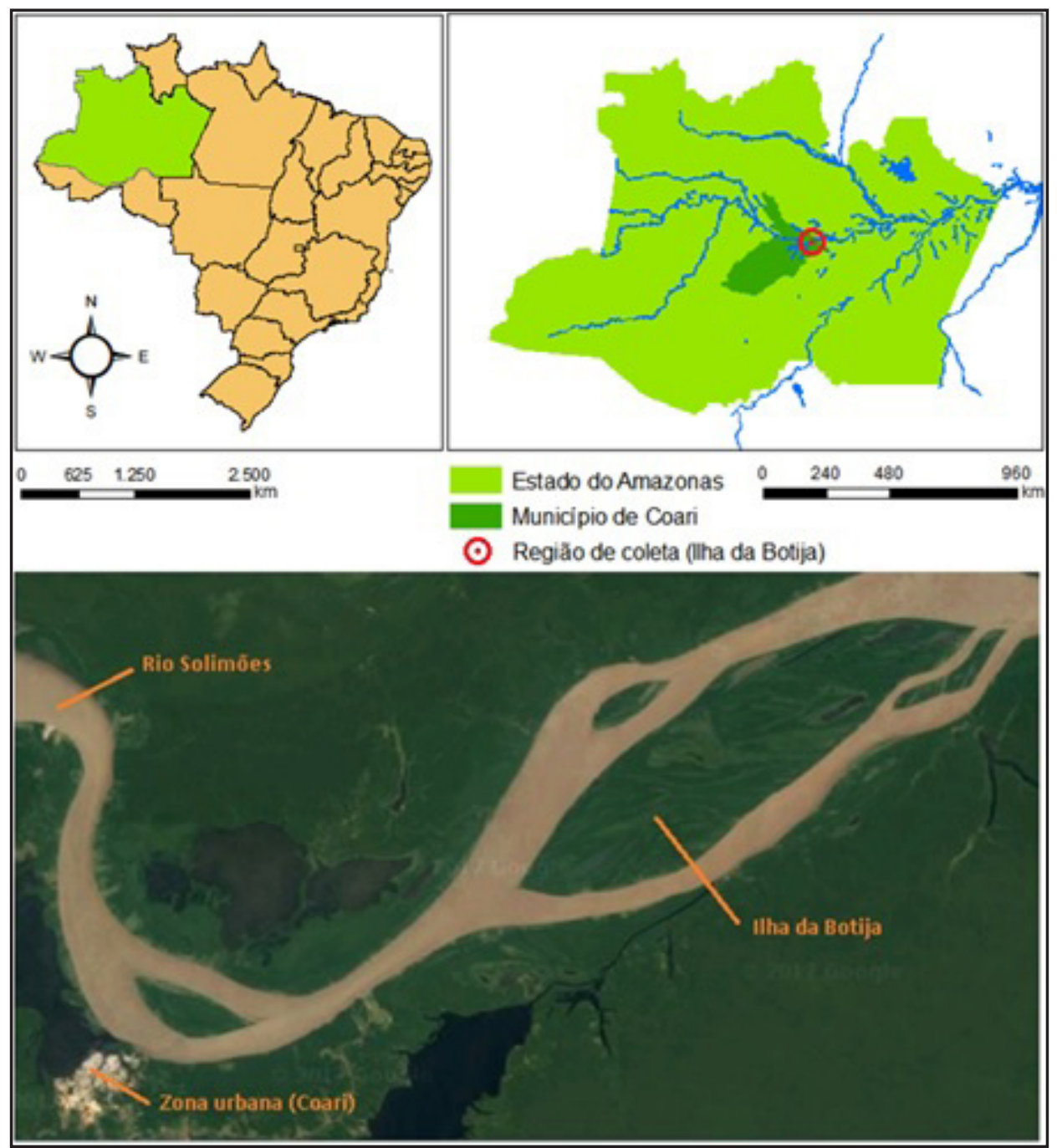

Fonte: Autores (2018) - Mapas acima; Google maps (2018) - Imagem abaixo

\section{Coleta de dados}

Na primeira etapa de coleta, para a medição do diâmetro dos troncos, foram isoladas duas áreas medindo aproximadamente 150 e $250 \mathrm{~m}^{2}$ (regiões 1 e 2). O tamanho das áreas foi delineado estimando-se que deveria haver entre 150 a 200 troncos mensuráveis em seu interior. Dentro dessas áreas foram medidas as espessuras (DAP nas árvores maiores e diâmetros um pouco antes da primeira ramificação nas menores) de todas as árvores com altura maior do que $0,50 \mathrm{~m}$ (região 1) e 1,50 m (região 2, na qual havia maior abundância de árvores maiores), com exceção dos indivíduos muito pequenos, cuja ramificação ocorria antes dessas alturas de corte.

$\mathrm{Na}$ segunda etapa de coleta, para as medidas dos galhos, foram escolhidos quatro 
espécimes da região 1: Neeacf. Mollis Spruceex J. A. Schmidt (pop. João-mole), Platonia insignis Mart. (pop. Bacurizeiro), Eugenia stipitata McVaugh (pop. Goiaba-araçá), Gustavia augusta L. (pop. Mucurão) e dois espécimes da região 2: Inga alba (Sw.) Willd. (pop. Ingá-ferro) e Bocageopsis multiflora (Mart.) R. E. Fr. (pop. Envireira). Prezando eficiência e tempo, alguns galhos primários (com todas as suas ramificações) foram escolhidos como amostras para os indivíduos maiores, o que não se mostra um problema, visto que a grande maioria das gerações ocorre em um único galho, suficiente para analisar a fractalidade da árvore. Foram então medidos, com fita métrica e paquímetro digital, as espessuras e comprimentos de cada galho, tomando cuidado de fazer uma rotulagem tal que a "ascendência" de cada um fosse conhecida. Algumas arbitrariedades foram inevitáveis, em especial na questão de decidir quando se tratava de uma bifurcação legítima ou de uma "multifurcação" (um galho "pai" com muitos "filhos"), o que afetaria a análise posterior da fractalidade. Os critérios utilizados na escolha foram a abundância das espécies, condições de acesso e as condições das espécies.

\section{Análise dos dados}

$\mathrm{Na}$ distribuição dos diâmetros dos troncos das regiões estudadas, fez-se um ajuste com lei de potência para observar se há realmente esse tipo de comportamento. Fez-se também uma comparação com dados da literatura para concluir se há ou não universalidade nesse comportamento. Com o objetivo de testar a hipótese das árvores "imitarem" sua região de entorno, foi feita a distribuição do diâmetro dos galhos para os espécimes coletados para comparação com as distribuições dos diâmetros dos troncos.

Para a análise da fractalidade, optou-se por uma abordagem diferente da usual (que é a dimensão fractal), baseada na estatística descritiva das relações entre diâmetros $(d)$ e comprimentos ( $l$ ) de galhos de gerações ( $k$ ) subsequentes ("pais" e "filhos"), respectivamente definidas conforme as Equações (2a) e (2b):

$$
\begin{aligned}
& \beta_{k} \equiv d_{k+1} / d_{k} \\
& \gamma_{k} \equiv l_{k+1} / l_{k}
\end{aligned}
$$

O argumento é que, pelo fato de um objeto fractal apresentar autossimilaridade, essas relações se manteriam as mesmas em fractais ideais. Sendo assim, pode-se imaginar que alguma medida de dispersão, como o coeficiente de variação $(C V)$, seja uma forma de estimar quantitativamente essa autossimilaridade, de modo que uma distribuição (de $\beta$ e $\gamma$ ) mais estreita (com menor dispersão) signifique que o padrão de ramificação da árvore em questão segue uma estrutura "mais fractal". A escolha do $C V$ parece adequada por permitir comparar variabilidades de conjuntos de dados com valores típicos muito diferentes. O uso do desvio padrão, por exemplo, só pode ser compreendido no contexto da média, e os valores médios de $\beta$ variam substancialmente de espécime para espécime. A dificuldade na estimativa do $C V$ se resume no fato do mesmo ser um estimador viesado e ter uma distribuição amostral bastante complexa (HENDRICKS; ROBEY, 1936). Uma aproximação para o intervalo de confiança (IC) para o CV foi proposta por Vangel (1996), e é dado pelas Equação (3):

$$
I C=\left\{C V\left[\left(\frac{\chi_{R}^{2}+2}{v+1}-1\right) C V^{2}+\frac{\chi_{R}^{2}}{v}\right]^{-1 / 2} ; C V\left[\left(\frac{\chi_{L}^{2}+2}{v+1}-1\right) C V^{2}+\frac{\chi_{L}^{2}}{v}\right]^{-1 / 2}\right\}
$$

onde: $v$ representa o número de graus de liberdade $(n-1) ; \chi_{R}^{2}$ e $\chi_{L}^{2}$ representam os valores críticos de $\chi^{2}$ nas caudas direita e esquerda, respectivamente, para o nível de significância pretendido. 


\section{Resultados}

\section{Distribuição dos diâmetros dos troncos}

As distribuições de frequência foram ajustadas por uma lei de potência em ambas as regiões (Figuras 2A e 2B).

Para inferir sobre a universalização do comportamento tipo lei de potência (e dos expoentes), fez-se uma comparação com a distribuição dos troncos em uma região do cerrado (SILVA JÚNIOR; SILVA, 1988), a qual pode ser vista na Figura 2C.

Figura 2 - Distribuições dos diâmetros dos troncos das regiões 1 (A) e 2 (B). (C) Distribuição dos diâmetros dos troncos em uma área do cerrado em Minas Gerais, Brasil. Os ajustes são do tipo lei de potência

Figure 2 - Distributions of trunk diameters in Regions 1 (A) and 2 (B). (C) Distribution of trunk diameters in a cerrado area in Minas Gerais, Brazil. The fittings are power-law type

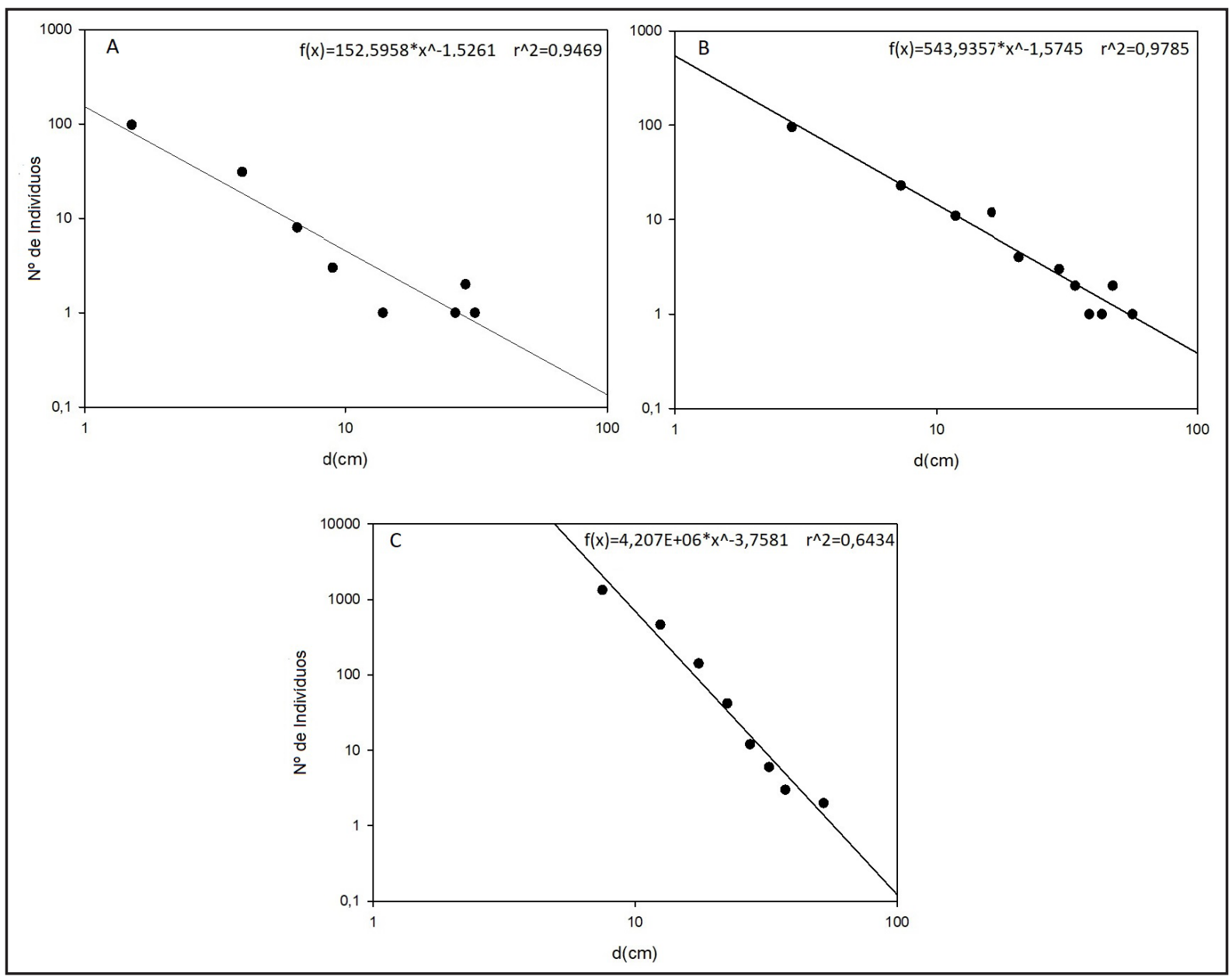

Fonte: (A) e (B): Autores (2018); (C) Silva Júnior e Silva (1988) 


\section{Distribuição dos diâmetros dos galhos}

As distribuições dos diâmetros dos galhos para os espécimes analisados nas regiões 1 (igapó) e 2 (várzea) são apresentados na Figura 3.

Figura 3 - Distribuições dos diâmetros dos galhos na região 1 (igapó), respectivamente para os espécimes de (A) Neea mollis (João-mole), (B) Platonia insignis (Bacurizeiro), (C) Eugenia stipitata (Goiaba-araçá) e (D) Gustavia augusta (Mucurão), e para a região 2 (várzea) (E) Inga alba (Ingá-ferro) e (F) Bocageopsis multiflora (Mart.) R. E. Fr.(Envireira). Os ajustes são do tipo lei de potência

Figure 3 - Distribution of branch diameters in region 1 (igapó), respectively for specimens of (A) Neeamollis (João-mole), (B) Platonia insignis (Bacurizeiro), (C) Eugenia stipitata (Goiaba-araçá) and (D) Gustavia augusta (Mucurão), and to region 2 (várzea) (E) Inga alba (Ingá-ferro) and (F)

Bocageopsis multiflora (Mart.) R. E. Fr.(Envireira). The fittings are power-lawtype

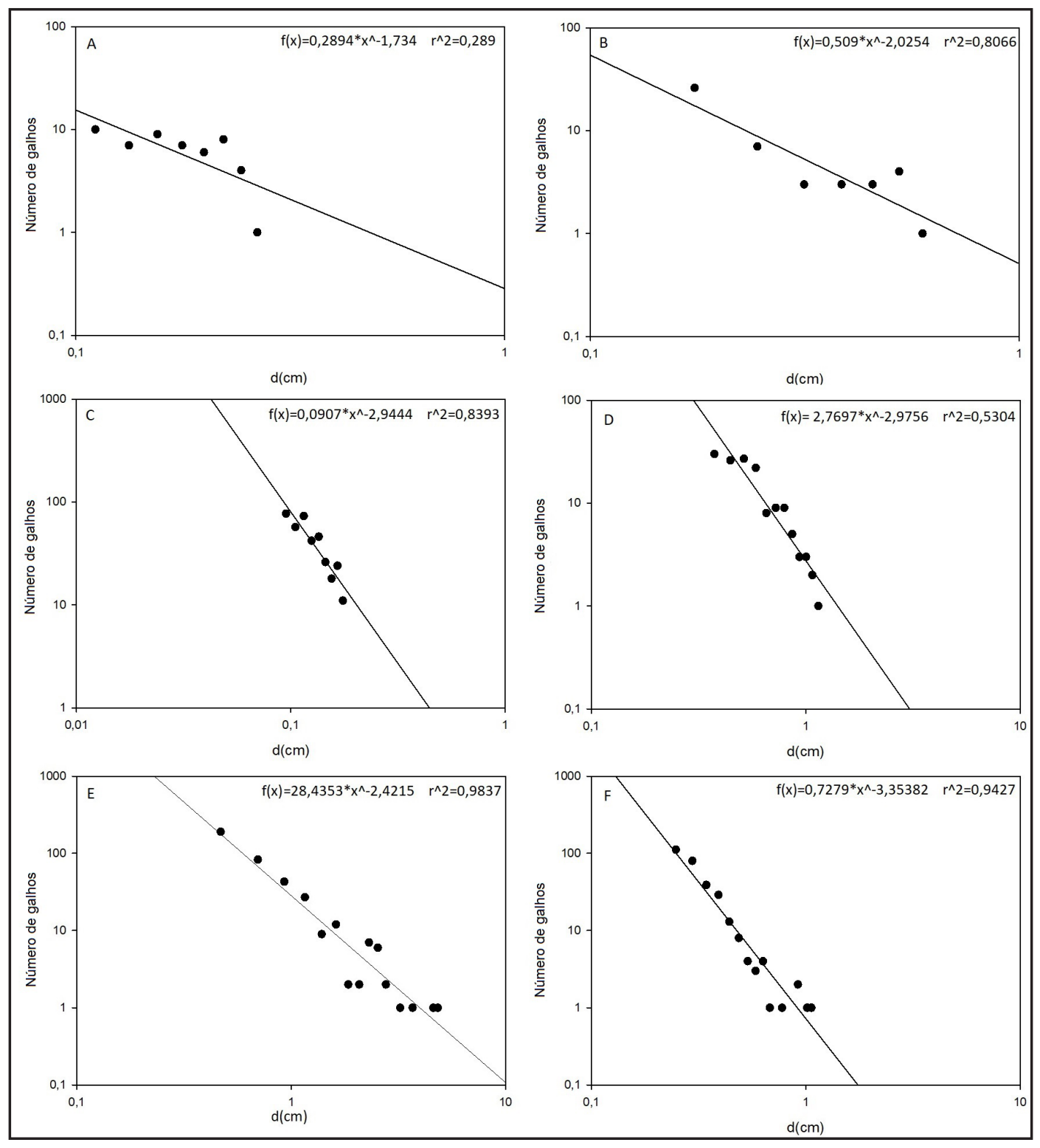

Fonte: Autores (2018) 


\section{Estatística descritiva das relações $\beta$ e $\gamma$}

Os histogramas para as relações entre diâmetros de galhos de gerações subsequentes ( $\beta$ ) são apresentados na Figura 4, em que pode ser notado o formato geral das distribuições para inferir qualitativamente acerca da fractalidade. Já o comportamento das relações entre os comprimentos $(\gamma)$ foi muito errático, além de conter muitos dados discrepantes. Por esses motivos, apenas $\beta$ foi utilizado nas análises deste trabalho.

Figura 4 - Histogramas das relações entre diâmetros de galhos de gerações subsequentes $(\beta)$ dos espécimes (em sequência) de (A) Neea mollis (João-mole), (B) Platonia insignis

(Bacurizeiro), (C) Eugenia stipitata (Goiaba-araçá), (D) Gustavia augusta (Mucurão), (E) Inga alba (Ingá-ferro) e (F) Bocageopsis multiflora (Mart.) R. E. Fr. (Envireira)

Figure 4 - Histogramsofrelationsbetweenbranchdiametersofsubsequentgenerations $(\beta)$ ofspecimens (in sequence) of(A) Neeamollis (João-mole), (B) Platonia insignis (Bacurizeiro), (C) Eugenia stipitata (Goiaba-araçá), (D) Gustavia augusta (Mucurão), (E) Inga alba (Ingá-ferro) and (F) Bocageopsis multiflora (Mart.) R. E. Fr. (Envireira)

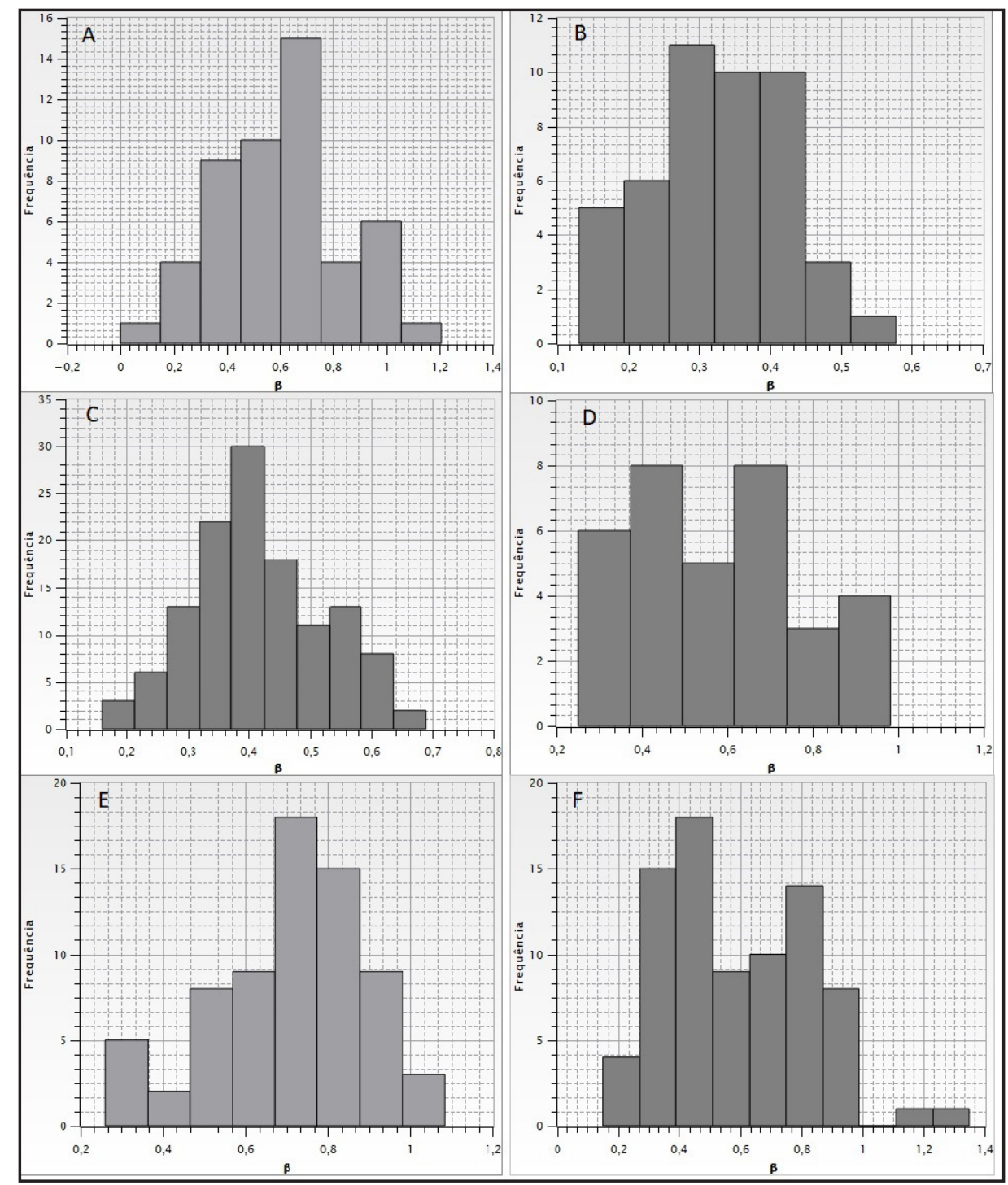

Fonte: Autores (2018) 
Pelo fato de os indivíduos analisados serem, em vários aspectos, substancialmente diferentes, se optou pelo cálculo do coeficiente de variação $(C V)$ para a comparação do caráter fractal (que chamado de fractalidade) das árvores. Ele é definido como a razão entre o desvio padrão e a média, i. e.: $C V=s / \bar{x}$, e os resultados estão apresentados na Figura 5.

Figura 5 - Coeficiente de variação $(C V)$ das relações entre diâmetros de galhos de gerações subsequentes $(\beta)$ para os diversos espécimes analisados. As barras representam os intervalos de confiança segundo a aproximação de Vangel (1996)

Figure 5 - Coefficient of variation $(C V)$ of relationships between branch diameters of subsequent generations $(\beta)$ for the various specimens analyzed. The bars represent confidence intervals according to Vangel's (1996) approximation

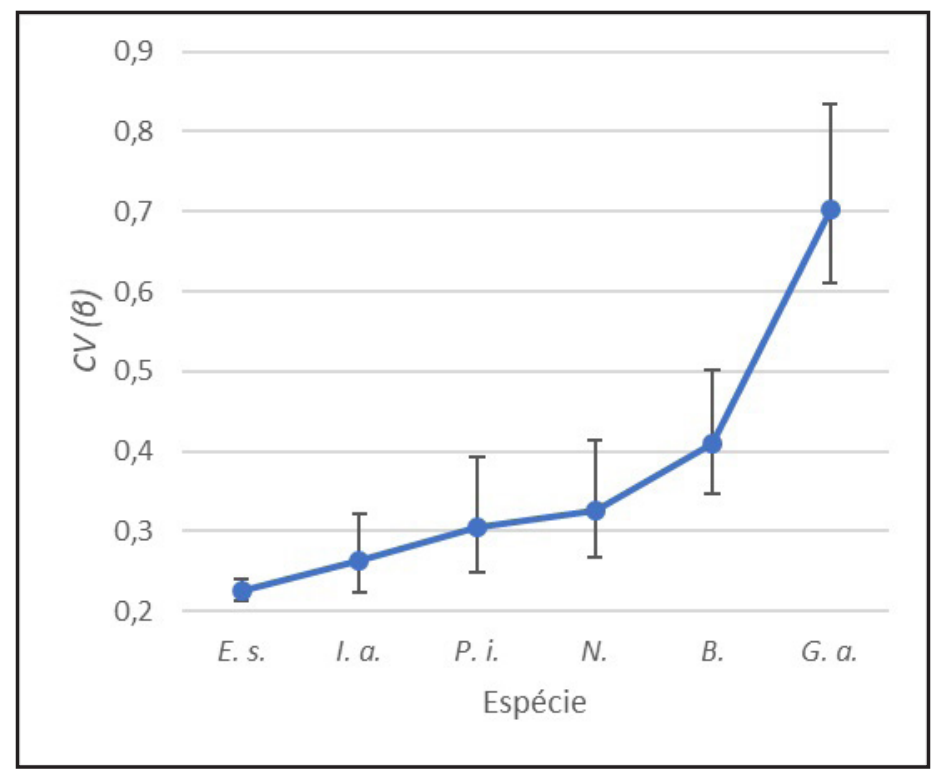

Fonte: Autores (2018)

\section{Discussão}

As distribuições dos troncos nas regiões analisadas seguem uma lei de potência com expoentes próximos de 1,5, afirmação que se pode ser sugerida com base nos valores de $R^{2}(0,9469$ e 0,9785 , respectivamente nas regiões 1 e 2). Com o objetivo de testar estatisticamente se a relação entre a frequência e o tamanho dos troncos é linear em uma escala logarítmica, foi utilizado o teste ANOVA. Foi testado, dentro de uma expansão utilizando polinômios ortogonais se o acréscimo de polinômios de ordem maior que 1 leva a um ajuste estatisticamente significativo. Nesse contexto, calculou-se a probabilidade (p) de que o acréscimo de polinômios de ordem maior que 1 não seja estatisticamente significativo. Para os dados da Figura 2, foram obtidos os seguintes resultados: para os troncos da região $1(\mathrm{~A}), \mathrm{p}=0,23$; para a região $2(\mathrm{~B}), \mathrm{p}=0,62$ e para os troncos do cerrado $(C), p=0,60$. Admitindo-se um nível de significância de $5 \%$, deve-se aceitar que a relação, em todos os casos, é linear. Esse comportamento era previsto teoricamente com um expoente igual a -2 (WEST; ENQUIST; BROWN, 2009), mas há divergências para esse valor em distribuições reais (MULLER-LANDAU et al., 2006; WANG et al., 2009). De fato, Enquist e Niklas (2011) mostraram, em um trabalho que incluiu um grande número de dados globais, que os expoentes de distribuição dos diâmetros dos troncos podem variar consideravelmente, com extremos próximos de 0,0 e -3,0. Além disso, parece haver uma leve dependência entre esses 
expoentes com a latitude. No trabalho realizado com árvores do Cerrado (Figura 2C), pode-se verificar uma maior discordância com o expoente teórico de -2 (o resultado foi próximo de $-3,7$ ), e até a ideia de universalidade do comportamento tipo lei de potência $\left(R^{2}=0,64\right)$, embora o teste estatístico não tenha permitido rejeitar a hipótese de linearidade.

No caso das distribuições dos diâmetros dos galhos, percebe-se um comportamento de lei de potência mais evidente para a região $2\left(R^{2}>0,9\right)$, e menos na região 1 , na qual os valores de $R^{2}$ variaram de aproximadamente 0,29 a 0,84 . Isso leva a sugerir que árvores maiores (mais maduras), caso dos espécimes analisados na região 2 , são mais propensas a seguir uma lei de potência. Tal fato sugere que a questão estrutural está mais ligada a esse comportamento matemático do que o aspecto hidrodinâmico da condução da seiva, pois galhos mais largos têm mais tecido estrutural (não condutor) do que galhos jovens. Realmente, há certa divergência na literatura acerca do que é predominante na formação geométrica das árvores e, embora o modelo WBE e outros (BEJAN, 1997) se baseiem na hipótese hidrodinâmica, outros trabalhos defendem a hipótese estrutural (JAMES; HARITOS; ADES, 2006; ELOY, 2011; KANG; DENNIS; MENEVEAU, 2011; RIAN; SASSONE, 2014), o que deixa o problema em aberto. Também foi realizado um teste (para a hipótese de linearidade) frente ao acréscimo de polinômios ortogonais até a ordem 4 . Os resultados ANOVA foram: João-mole $(\mathrm{A}), \mathrm{p}=0,10$; Bacurizeiro $(\mathrm{B}), \mathrm{p}=0,85$; Goiaba-araçá $(\mathrm{C}), \mathrm{p}$ $=0,050$; Mucurão (D), $p=0,0038$; Ingá-ferro (E), $p=0,33$; Envireira $(F), p=0,036$. Assim, deve-se admitir imediatamente que os dados referentes a João-mole, Bacurizeiro, Goiaba-araçá e Ingáferro são lineares. Quanto aos dados referentes à Envireira, o teste estatístico demonstra que o resultado foi significativamente influenciado pelos pontos com frequência igual a 1 , ou seja, as medidas de diâmetros que encontraram apenas um resultado. Qualquer um desses pontos que seja retirado do conjunto de dados, leva a $\mathrm{p}>0,05$. $\mathrm{Na}$ análise estatística, não foram dados pesos diferenciados aos pontos correspondentes a diferentes frequências. Já o Mucurão corresponde aos dados que mais se diferenciam da linearidade. Contudo, a análise com polinômios ortogonais demonstra que existe uma componente quadrática significativa (na verdade isso pode ser observado visualmente no gráfico). A probabilidade frente à introdução de polinômios de ordem maior que 3 é 0,86. Entretanto, é difícil acreditar que a hipótese da existência real de uma relação quadrática seja cientificamente plausível. Nesse caso, também a análise estatística é influenciada pelo ponto que corresponde à frequência de 1 evento. Além disso, o extremo superior da curva (dados de alta frequência e pequeno diâmetro), pode ter sido influenciado pela consistência física dos dados, ou seja, limitação da frequência pelo fato de que galhos com dimensão menor que um certo limite não tenham consistência física, ou seja, se quebrem com facilidade. Diante disso, a hipótese de linearidade é a que se apresenta mais consistente do ponto de vista científico em todos os casos.

A questão da aplicabilidade do modelo WBE nos indivíduos estudados merece uma discussão detalhada. Primeiramente, é preciso expor que algumas espécies têm o processo de ramificação dominado por bifurcações, outras por multifurcações (um galho "pai" com muitos galhos "filhos"), e algumas não têm uma dominância definida, tendo um comportamento misto. O modelo WBE estabelece a relação matemática destas $n$-furcações: (WEST; BROWN; ENQUIST, 1997). Assim, se o modelo se aplica, deveriam aparecer picos na distribuição de $\beta$ ao redor de (ou 0,707 ) nas árvores de comportamento bifurcado, picos em regiões anteriores no comportamento multifurcado e ao menos dois picos pronunciados no caso misto. Essa lógica é consistente com a análise apresentada na Figura 4, para as espécies analisadas, visto que os espécimes de Neea mollis (João-mole), Eugenia stipitata (Goiaba-araçá) e Inga alba (Ingá-ferro) possuem comportamento dominado por bifurcações, o Platonia insignis (Bacurizeiro) tem um comportamento multifurcado, praticamente sem bifurcações, e o Gustavia augusta (Mucurão) e Bocageopsis multiflora (Envireira) possuem um comportamento misto, com bifurcações e multifurcações. No entanto, chama atenção o espécime de Eugenia stipitata, que embora tenha um único pico relevante na distribuição de $\beta$, o mesmo é pronunciado em torno de 0,4 , valor não previsto pelo modelo. 
Quanto à estimativa de autossemelhança (fractalidade), a Figura 5 sugere quais espécimes seguem um padrão mais próximo a um fractal, pois quanto menor for $C V$, maior será, em tese, a fractalidade da árvore em questão, no sentido de que as relações entre os diâmetros dos galhos "filhos" e "pais" são semelhantes ao longo das gerações de ramificação. Por essa razão, acreditase que essa é uma medida razoavelmente adequada para comparar autossemelhança, o que é, em síntese, o que define uma estrutura fractal. Nas amostras com $n$ menor, houve intervalos de confiança muito grandes e sobrepostos, o que impede de fazer uma classificação objetiva nesse quesito. Por fim, os resultados obtidos sugerem não haver uma clara conexão entre comportamentos do tipo lei de potência e fractalidade, pois nem sempre um bom valor de $R^{2}$ para o ajuste de lei de potência significou um baixo valor (relativamente) de $C V$.

Cabe aqui uma importante discussão acerca do papel que a geometria exerceu (e exerce) na trajetória evolutiva das árvores. As sugestões apresentadas pelas teorias de WBE, Eloy ou Bejan se apresentam como promissoras e ajudam a elucidar uma série de questionamentos, mas talvez se excedam por quererem partir de princípios muito universais. Provavelmente não foi um único motivo e uma única etapa na evolução que trouxe as árvores ao que são hoje (GUILHERME, 2017). Pode-se pensar na questão das árvores monocotiledôneas (Arecaceas ou palmeiras) versus eudicotiledôneas (quase a totalidade das outras angiospermas), pois as diferenças são muitas. Pode-se sugerir que as eudicotiledôneas têm um sucesso evolutivo relativamente maior, já que são muito mais numerosas em número de exemplares e diversidade de espécies, o que faz todo o sentido pelo seu padrão de ramificação e distribuição de nutrientes, segundo a ótica da teoria de WBE. Mas se esse fosse o único princípio norteador da evolução das árvores, as palmeiras sequer existiriam, uma vez que há indícios de que elas são mais derivadas (menos primitivas) que grande parte das eudicotiledôneas, mostrando que a complexidade estrutural não parece ser tão crucial, ao menos em regiões quentes, baixas e úmidas, nas quais as palmeiras são abundantemente encontradas (SALM et al., 2007). De fato, nessas regiões, elas parecem ser muito bem-sucedidas, por uma série de fatores, principalmente climáticos (WINDSOR-COLLINS et al., 2007). Retornando agora à questão das árvores de padrão ramificado, têm-se algumas questões a elucidar. Como as eudicotiledôneas são muito dominantes, é de se supor que ter um tronco mais espesso (e energeticamente "caro") que sustente uma grande estrutura de ramificação deva valer a pena. As principais teorias são baseadas na otimização de transporte de nutrientes como se conjecturava desde a década de 1970 (LEOPOLD, 1971), quando foi proposto que uma estrutura ramificada hierarquizada era mais eficiente, em especial no sentido de minimizar o percurso total de fluidos. Vale frisar que estrutura ramificada (hierárquica) na natureza não significa necessariamente uma estrutura fractal, como observado por alguns trabalhos (AVNIR et al., 1998; BEJAN, 2000; HALLEY et al., 2004). No entanto, como já citado, há estudos que focam no aspecto estrutural e mecânico das árvores (RIAN; SASSONE, 2014). Por exemplo, há evidências de que o padrão fractal faça com que haja uma melhor distribuição de forças ao longo da árvore, algo muito necessário em situações de fortes ventos (ELOY, 2011). Mas se observada, uma estrutura ramificada se apresenta como muito eficiente justamente para se autossustentar, e o argumento pode ser cíclico. Isto é, uma árvore seria ramificada de forma aproximadamente fractal para otimizar a distribuição de nutrientes e resistir a estresses mecânicos, duas coisas dispensáveis se a árvore não tivesse esse tipo de estrutura dispendiosa. Então outros motivos levam as árvores a adotarem seus formatos, e o mais evidente de todos e que possivelmente seja o mais importante é a eficiência na captação de luz solar (HONDA; FISHER, 1978). Outros motivos podem ser conjecturados, como a multiplicidade de meristemas, o que permite à planta sobreviver e voltar a crescer mesmo após perder vários brotos, seja por ação de herbívoros ou eventos meteorológicos, diferentemente do que ocorre com as monocotiledôneas e seu o meristema apical. Também pode ser interessante, do ponto de vista reprodutivo, ter frutos (ou esporângios) es palhados ao longo de grandes copas, o que facilita evidentemente a dispersão de sementes na área ao redor do tronco.

Resumidamente, parece ser enfático que os formatos (e sua diversidade) das árvores resultam de uma espécie de ajuste (ou "negociação") entre seus diferentes objetivos (THOMAS,

Ci. Fl., Santa Maria, v. 30, n. 4, p. 1061-1074, out./dez. 2020 
2014). A natureza desse ajuste pode ser difícil de apontar, mas algumas generalizações podem ser feitas. Por exemplo, coníferas de grande latitude e/ou altitude devem sua forma à necessidade de reduzir o acúmulo de neve e de interceptar o máximo de luz solar, pois em grandes latitudes o sol tende a estar posicionado mais próximo do horizonte que do azimute, o que explica o fato de florestas de coníferas serem bastante escuras em imagens de satélite (THOMAS, 2001).

\section{Conclusão}

Após tudo que foi discutido, pode-se tentar responder às três questões colocadas anteriormente. 1) A distribuição de frequência dos troncos sugere que há sim uma relação do tipo lei de potência (livre de escala) para a distribuição dos diâmetros dos troncos nas áreas de estudo. Uma curva de ajuste do tipo lei de potência teve boa concordância $\left(R^{2}>0,94\right)$, com expoentes em torno de -1,53 e 1,57 (igapó e várzea). Contudo, não se pode afirmar a universalidade do comportamento tipo lei de potência na distribuição dos troncos, uma vez que um valor encontrado em um estudo dentro do bioma sul-americano resultou em um expoente de $-3,7$ e $R^{2}$ comparativamente menor, de 0,64. Em trabalho do próprio Enquist com um grande número de dados globais, se conclui que a distribuição dos diâmetros dos troncos apresenta expoentes que podem variar consideravelmente, com extremos próximos de 0,0 e -3,0. 2).Pode-se sugerir que a distribuição de frequência dos diâmetros dos galhos seguiu uma lei de potência $\left(R^{2}>\right.$ 0,80) nos espécimes de Platonia insignis (Bacurizeiro), Eugenia stipitata (Goiaba-araçá), Inga alba (Ingá-ferro) e Bocageopsis multiflora (Envireira), o que não ocorreu nos espécimes de Neea mollis (João-mole) e Gustavia augusta (Mucurão) $\left(R^{2}<0,80\right)$. A semelhança com a distribuição dos troncos foi no formato (para os espécimes que seguiram a lei de potência), mas não nos expoentes encontrados, que variaram de $-3,3$ a -2,0. Esses valores não permitem afirmar que as espécies de uma floresta repetem os padrões geométricos dessa mesma floresta, ou seja, nem sempre a ramificação de uma espécie "imita" a área que ela está inserida (em termos de distribuição e diâmetros). Talvez isso possa acontecer em média, mas mais estudos precisariam ser feitos, com um grande número de indivíduos. 3) Sendo o fractal uma entidade matemática idealizada, assumir a hipótese de fractalidade para objetos reais pode ser discutível. O que se tentou fazer foi uma análise comparativa com base em argumentos quantitativos que reflitam algum grau de autossimilaridade a partir do coeficiente de variação de $\beta$. Pela grande sobreposição dos intervalos de confiança, não é possível classificar estatisticamente todas as espécies por ordem de fractalidade. No entanto, fica claro que a árvore mais autossemelhante foi a Eugenia stipitata, e a menos foi a Gustavia augusta, e algum grau absoluto de fractalidade pode ser atribuído a todos os espécimes, pelas prevalências de $\beta$ em alguns ranges de valores. Esse tipo de comportamento pode ser importante na otimização de transporte de fluidos, como sugere o modelo WBE, mas uma grande estrutura de ramificação, tenha ela elementos de autossimilaridade ou não, não parece fazer sentido por si só. Também não parece haver um único princípio que possa explicar o formato das árvores, e os modelos devem levar isso em consideração. A hipótese da fractalidade como o fator preponderante no formato pode ser uma inferência muito forte e que desconsidera o sucesso evolutivo das espécies que não seguem esse padrão (como as palmeiras). Outras questões como resistência a estresse e a distúrbios eventuais, capacidade de recuperação e mudanças metabólicas (como o tipo de fotossíntese) podem ser fundamentais.

\section{Agradecimentos}

Gostaríamos de agradecer à CAPES pelo financiamento e aos membros da comunidade ribeirinha da "Ilha da Botija" pelo apoio logístico e hospitalidade, em especial ao Prof. Valcileno Pinheiro da Silva. 


\section{Referências}

AVNIR, D. et al. Is the Geometry of Nature Fractal? Science, Washington, v. 279, p. 39-40, jan. 1998.

BARTHOLOMEW, G. A. A matter of size; An examination of endothermy in insects and terrestrial vertebrates. In: HEINRICH, B. (ed.). Insect thermoregulation. New York: Wiley, 1981.

BEJAN, A. Constructal-theory network of conducting paths for cooling a heat generating volume. International Journal of Heat Mass Transfer, Amsterdam, v. 40, p. 799-816, mar. 1997.

BEJAN, A. Shape and structure, from engineering to nature. Cambridge: Cambridge University Press, 2000.

BRODY, S.; PROCTER, R. C.; ASHWORTH, U. S. Basal metabolism, endogenous nitrogen, creatinine and neutral sulphur excretions as functions of body weight. University of Missouri Agricultural Experiment Station Research Bulletin, Jefferson City, v. 220, p. 1-40, 1934.

CALDER, W. A. Size, function, and life history. Cambridge: Harvard University Press, 1984.

ELOY, C. Leonardo's rule, self-similarity and Wind-induced stresses in trees. Physical Review Letters,College Park, v. 107, p. 258101, dec. 2011.

ENQUIST B. J.; NIKLAS, K. J. Invariant scaling relations across tree-dominated communities. Nature, Londres, v. 410, p. 655-660, apr. 2001.

FISCH, G. Uma revisão geral sobre o clima da Amazônia. Acta Amazonica, Manaus, v. 28, n. 2, p. 101-101, jun. 1998.

GUILHERME, A. P. Abordagem teórico-experimental das relações de escala e fractalidade: uma aplicação do modelo "WBE" em área de várzea da floresta amazônica. 2017. Tese (Doutorado em Física Ambiental) - Universidade Federal de Mato Grosso, Instituto de Física, Cuiabá, 2017. Disponível em: http://www.pgfa.ufmt.br/index.php/br/utilidades/teses/368-adriano-pereiraguilherme/files

HALLEY, J. M. et al. Uses and abuses of fractal methodology in ecology. Ecology Letters, Paris, v. 7, p. 254-271, 2004.

HENDRICKS, W. A.; ROBEY, K. W. The Sampling Distribution of the Coefficient of Variation. The Annals of Mathematical Statistics, Beachwood, v. 7, n.3, p. 129-132, 1936. DOI:10.1214/ aoms/1177732503

HONDA, H.; FISHER, J. B. Tree Branch Angle: Maximizing Effective Leaf Area. Science, v. 199, p. 888-890, feb. 1978.

JAMES, K. R.; HARITOS, N.; ADES, P. K. Mechanical stability of trees under dynamic loads. American Journal of Botany, San Luis, v. 93, n. 10, p. 1522-1530, 2006.

KANG, H. S.; DENNIS, D.; MENEVEAU, C. Flow over fractals: drag forces and near wakes. Fractals, Singapura, v. 19, n. 4, p. 387-399, 2011.

KLEIBER, M. Body size and metabolism. Hilgardia, Berkeley, v. 6, p. 315-353, 1932.

KOZŁOWSKI, J.; KONARZEWSKI, M. Is West, Brown and Enquist's Model of Allometric Scaling Mathematically Correct and Biologically Relevant? Functional Ecology, London, v. 18, n. 2, p. 283-289, apr. 2004.

LEOPOLD, L. B. Trees and Streams: The Efficiency of Branching Patterns. Journal of theoretical Biology, Amsterdam, v. 31, p. 339-354, 1971.

MULLER-LANDAU, H. C. et al. Comparing tropical forest tree size distributions with the 
predictions of metabolic ecology and equilibrium models. Ecology Letters, Paris, v. 9, p. 589602, 2006.

NAÇÕES UNIDAS. Influência humana no aquecimento global é evidente, alerta novo relatório do IPCC. [S. l.], 2014. Disponível em: https://nacoesunidas.org/influencia-humana-noaquecimento-global-e-evidente-alerta-novo-relatorio-do-ipcc/. Acesso em: 22 jun. 2016.

PETERS, R. H. The ecological implications of body size. Cambridge: Cambridge University Press, 1983.

RIAN, I. M.; SASSONE, M. Tree-inspired dendriforms and fractal-like branching structures in architecture: a brief historical overview. Frontiers of Architectural Research, Beijing, v. 3, p. 298-323, 2014.

RUBNER, M. Uber den Einfluss der Korpergrosse auf Stoff-und Kraftwechsel, Zeitscrift fur Biologie, Munique, v. 19, p. 535-562, 1883.

SALM, R. et al. Cross-scale determinants of palm species distribution. Acta Amazonica, Manaus, v. 37, n. 1, p. 17-26, 2007.

SCHMIDT-NIELSEN, K. Scaling: why is animal size so important? Cambridge: Cambridge University Press, 1984.

SERVIÇO FLORESTAL BRASILEIRO. Florestas do Brasil em resumo- 2010: dados de 20052010. Brasília, 2010. 152 p. Disponível em: http://www.mma.gov.br/estruturas/sfb/_arquivos/

SILVA JÚNIOR, M. C.; SILVA, A. F. Distribuição dos diâmetros dos troncos das espécies mais importantes do cerrado na estação florestal de experimentação de Paraopeba (EFLEX)-MG. Acta Botanica Brasilica, Brasília, v. 2, n. 1/2, p. 107-126, 1988.

THOMAS, P. The shapes of trees: a matter of compromise. Arnoldia, [s. l.], v. 61, n. 1, p. 14-21, 2001.

THOMAS, P. The shape of trees. In: TREES: Their Natural History. 2nd ed. Cambridge: Cambridge University Press, 2014.

VANGEL, M. G. Confidence Intervals for a Normal Coefficient of Variation. The American Statistician, Boston, v. 50, n. 1, p. 21-26, 1996.

WANG, X. et al. Tree size distributions in an old-growth temperate forest. Oikos, Lund, v. 118, p. 25-36, 2009.

WEST, G. B.; BROWN, J. H.; ENQUIST, B. J. A general model for the origin of allometric scaling laws in biology. Science, Washington, v. 276, p. 122-126, apr. 1997.

WEST, G.; ENQUIST, B.; BROWN, J. The fourth dimension of life: fractal geometry and allometric scaling of organisms. Science, Washington, v. 284, p. 1677-1679, jun. 1999.

WEST, G.; ENQUIST, B.; BROWN, J. A general quantitative theory of forest structure and dynamics. PNAS, Washington, v. 106, n. 17, p. 7040-7045, apr. 2009.

WINDSOR-COLINS, A. et al. The palm - a model for success? In: DESIGN and Information in Biology. Billerica: WIT Press, 2007. p. 303-326. 